\title{
Toll-Like Receptor 4 Stimulation Triggers Crescentic Glomerulonephritis by Multiple Mechanisms Including a Direct Effect on Renal Cells
}

Angela Giorgini, Heather J. Brown, Steven H. Sacks, and Michael G. Robson

From the Medical Research Council Centre for Transplantation, King's College London School of Medicine, London, United Kingdom

A role for toll-like receptor 4 (TLR4) has been suggested in previous studies of glomerulonephritis, but the complex integration of these effects has not been explored. To separate effects on the innate and adaptive immune responses, we use the autologous nephrotoxic nephritis model with two disease induction protocols. First, we give a TLR4 ligand at the time of immunization and show the effects are mediated via TLR4 by comparing wild-type and TLR4-deficient mice. In wild-type mice histological measures of disease and serum creatinine are all at least twice as high as TLR4-deficient mice, due to an enhanced immune response to the nephritogenic sheep IgG. Second, we stimulate TLR4 later in the course of disease development and construct four groups of bone marrow chimeric or sham chimeric mice to study the role of TLR4 on bone marrow or renal cells. The most striking finding is that renal cell TLR4 stimulation increases glomerular crescent formation, with a mean of $21 \%$ and $25 \%$ in the two groups of mice with renal cell TLR 4 compared with $0.1 \%$ and $0.6 \%$ in the two groups without, with differences mirrored by changes in serum creatinine. These findings, in a single disease model, illustrate that TLR4 stimulation triggers crescentic glomerulonephritis by effects on both the adaptive and innate immune response, with a crucial direct effect on renal cells. (Am J Pathol 2010, 177:644-653; DOI: 10.2353/ajpath.2010.091279)

Toll-like receptors (TLRs) are a family of pattern recognition receptors that play a central role in the cellular response to microbes. They are likely to be central to any link between glomerulonephritis and infection. It was first discovered that TLR4 recognized lipopolysaccharide
(LPS), otherwise known as Gram-negative endotoxin. This was found when two mouse strains were observed to have mutations in the t/r 4 gene. ${ }^{1}$ Gene targeting has been used to construct TLR4-deficient mice, which has confirmed the role of TLR4 in the recognition of LPS. ${ }^{2}$ Subsequently, other members of the TLR family have been shown to recognize a range of microbial components and 11 mammalian TLRs have been described. ${ }^{3}$ As well as leukocytes, TLRs are also widely expressed on nonbone marrow-derived cells. ${ }^{4}$ These tissue-cell TLRs have been shown to contribute to disease in several nonrenal experimental models ${ }^{5,6}$ in addition to models of renal tubulointerstitial disease. ${ }^{7-10}$

TLR2 and TL4 use the adaptor molecule TIRAP to activate the Myd88 dependent signaling pathway that includes IRAK4 and TRAF6. In addition, TLR4 activates Myd88 independent pathways via TRIF. Hence one would expect similarities in the effects of these two receptors on disease severity. We have recently established that stimulation of TLR2 and TLR4 can trigger disease in the heterologous phase of nephrotoxic nephritis. ${ }^{11,12}$ This is a model of neutrophil-mediated acute glomerular inflammation induced by passively administered glomerular binding antibody. ${ }^{13}$ For both TLR2 and TLR4, we demonstrated that stimulation of renal cell TLRs played an important role. The autologous phase of nephrotoxic nephritis is due to an immune response to the foreign glomerular bound antibody, which acts as a planted antigen. ${ }^{14}$ It is a more clinically relevant model of glomerulonephritis as the pathology shows proliferation, macrophage and T cell infiltration, and thrombosis and crescent formation, ${ }^{15}$ which are all features found in human disease. We have recently demonstrated that TLR2 ligands given at the time of priming to the planted antigen

Supported by Guy's and St Thomas' Charity and the Wellcome Trust.

Accepted for publication April 23, 2010.

Supplemental material for this article can be found on http://ajp. amjpathol.org.

Address reprint requests to Michael G. Robson, Ph.D., MRCP, MRC Centre for Transplantation, 5th Floor Tower Wing, Great Maze Pond, King's College London School of Medicine, Guy's Hospital, London SE1 9RT, United Kingdom. E-mail: michael.robson@kcl.ac.uk. 
can exacerbate disease in this model by modulating the nephritogenic immune response. ${ }^{16}$ In addition to our own studies, Fu et $\mathrm{al}^{17}$ also gave endotoxin and other TLR ligands to mice at the same time as nephrotoxic antibody. However, in this work there was no measurement of the immune response to the nephritogenic antigen sheep $\mathrm{IgG}$, and so the relative roles of effects on the adaptive immune response or on innate mechanisms were not clear.

The current study had two main aims. First, we wanted to assess whether stimulation of TLR4 at the time of priming could cause crescentic glomerulonephritis by augmenting the adaptive immune response to sheep IgG, as we had shown for TLR2. Second, we planned to study the effect of TLR4 stimulation on crescentic glomerulonephritis in the context of an established nephritogenic immune response, including an assessment of the role of TLR4 on renal cells. This would provide an indepth understanding of the effects of TLR4 stimulation on both innate and adaptive immune mechanisms in a single clinically relevant model of glomerulonephritis.

\section{Materials and Methods}

\section{Mice}

Wild-type C57BL6 mice were from Harlan UK. TLR4deficient mice were kindly provided by S. Akira. These were backcrossed seven generations to C57BL/6. All experiments were performed according to UK home office and local regulations.

\section{Induction of Glomerulonephritis}

Nephrotoxic serum (NTS) was raised in sheep as described, ${ }^{11}$ and two disease inducing protocols were used (LPS at priming, and LPS given later, denoted protocols $A$ and $B$, respectively). Sheep IgG used for pre-immunization was prepared in house from normal sheep serum by using DEAE sepharose with purity more than $90 \%$ on SDS-polyacrylamide gel electrophoresis. In protocol A, mice were immunized intraperitoneally with $200 \mu \mathrm{g}$ of normal sheep IgG that was given with $80 \mu$ l of aluminum hydroxide gel (Sigma, Poole, UK) in $310 \mu$ of PBS (endotoxin free from Sigma), with $10 \mu \mathrm{g}$ of highly purified LPS from Escherichia coli R515 (581-007-L002; Alexis Biochemicals, Nottingham, UK ). All of these components were given as a single intraperitoneal injection. Five days later, mice received an injection of $160 \mu$ l of NTS via the tail vein to induce disease. In protocol B, disease was induced with $10 \mu \mathrm{l} / \mathrm{g}$ of serum intravenously. At days 5 and 7, $10 \mu \mathrm{g}$ LPS was given intraperitoneally in $400 \mu \mathrm{l}$ PBS, with PBS alone as a control. These protocols are illustrated in Figure 1. Endotoxin levels were measured by using a chromogenic kinetic Limulus Amoebocyte Lysate assay (Cambrex Bioscience, Verviers, Belgium). NTS used in all experiments contained $<0.5 \mathrm{eU} / \mathrm{ml}$. Eighty microliters aluminum hydroxide contained $<0.004 \mathrm{eU}$. The 200- $\mu$ g sheep IgG used for pre-immunization contained $<0.0025 \mathrm{eU}$. Only mice surviving to the experi-

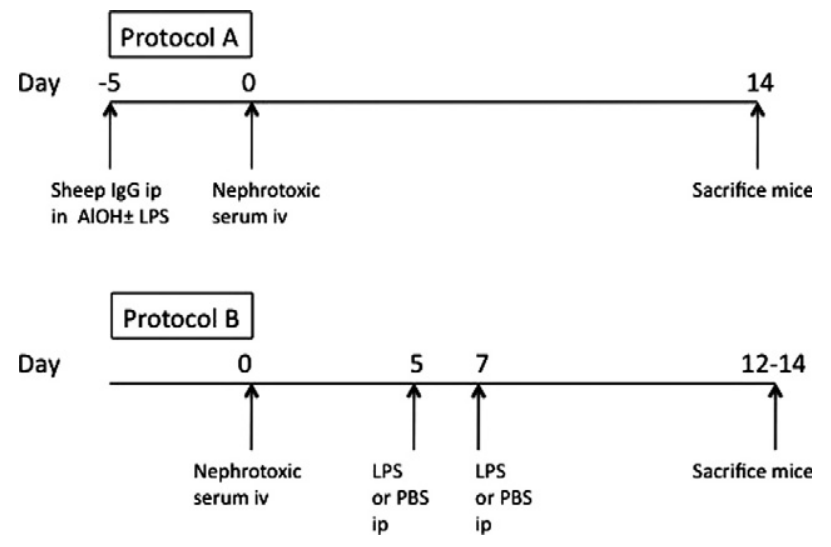

Figure 1. Overview of disease induction protocols. Glomerulonephritis was induced by using two protocols. In protocol A, LPS or control was given at the time of immunization with sheep $\operatorname{IgG}$ in aluminum hydroxide. In the second protocol, our aim was to have no effect on the immune response to sheep IgG, and LPS was given after NTS injection. The protocols are described in detail in Materials and Methods.

ment end were included in the analysis. For the experiment depicted in Figures 2 to 4, one wild-type mouse was sacrificed on day 6 due to ill-health and one on day 7 , with one TLR4-deficient mouse sacrificed on day 12, and the remainder on day 14 . In addition, one wild-type mouse was not placed in the metabolic cage at the end of the experiment due to ill health. For the experiment in Figures 5 to 7, two mice given LPS were sacrificed on day 10 due to ill health, and the remainder on day 12. For the experiment in Figures 8 to 10, one wild-type $\rightarrow$ wild-type mouse was sacrificed on day 12 due to ill health, and the remainder on day 14 (with blood and urine not obtained from one wild-type $\rightarrow \mathrm{TLR}^{-1-}$ mouse, which was ill on day 14).

\section{Assessment of Disease}

Disease was assessed as previously described. ${ }^{16}$ In brief, crescents and thrombosis were scored on samples fixed in Bouin's solution and stained with PAS. For thrombosis, 50 glomeruli were each scored out of 4. Neutrophils were identified by their characteristic nuclear morphology on PAS stained sections. $\mathrm{CD}^{+} 8^{+}$and $\mathrm{CD} 4^{+}$cells were detected on phosphate-lysine periodate fixed sections, with anti-CD68 (clone FA11; Serotec, Oxford, UK) and anti-CD4 (clone L3T4; BD Pharmingen, Oxford, UK), respectively. For the experiments in wild-type mice, the avidin-biotin complex method was used with a biotinylated mouse anti-rat (Jackson's Immunoresearch, Westgrove, PA) and avidin-biotin complex kit (Vectorlabs, Peterborough, UK). For the chimera experiments, we changed to an immunofluorescence-based method. Detection was with fluorescein isothiocyanate conjugated mouse anti-rat IgG (Jacksons Immunoresearch), followed by Alexa Fluor 488 conjugated goat anti-fluorescein isothiocyanate (Molecular Probes, Paisley, UK). All histological scoring was done without knowledge of the sample identity. Albuminuria was measured by radial immunodiffusion by using rabbit anti-mouse albumin (Biogenesis, Poole, UK) as described previously. ${ }^{11}$ Normal C57BL/6 
A
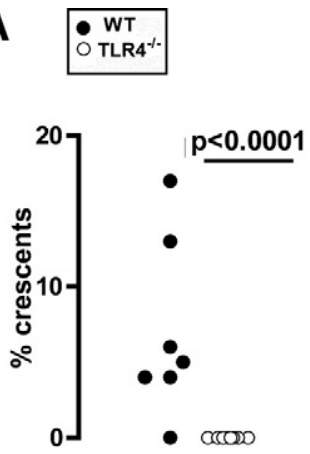

C

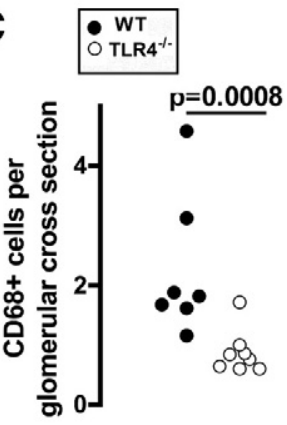

E

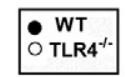

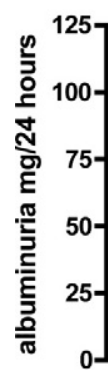

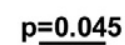

D

$\mathbf{F}$

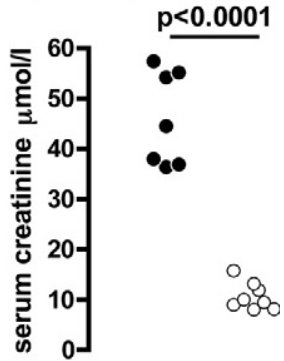

Day 14
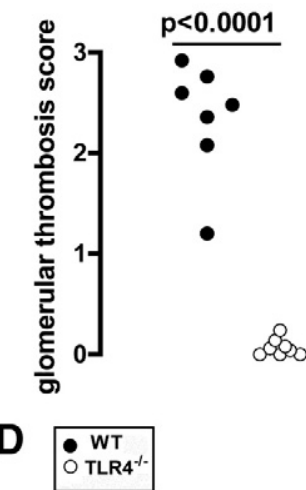

$\$ \infty$
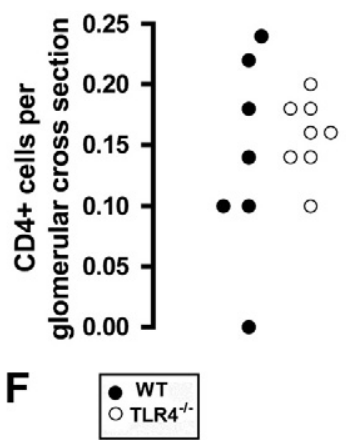

B $\begin{gathered}0 T \\ 0 \text { TLR4 }\end{gathered}$

0.0001

080

Figure 2. TLR 4 stimulation at immunization exacerbates crescentic glomerulonephritis. Wild-type and TLR4-deficient mice were pre-immunized with sheep IgG and LPS and disease was induced as described in protocol A. Disease parameters were assessed at day 14 (albuminuria at day seven as well). A-D: In wild-type, compared with TLR4-deficient mice, there were significantly more crescents, thrombosis, and glomerular $\mathrm{CD}_{6} 8^{+}$macrophages, but no difference in glomerular $\mathrm{CD}^{+}$cell numbers. E: Albuminuria was not different at day seven and higher in TLR4-deficient mice at day 14 . F: Serum creatinine (day 14) was higher in wild-type mice. Each point is an individual mouse $n=7$ (wild-type) and $n=8$ (TLR4-deficient). Mice not surviving to day 14 were not included in the analysis and are listed in Materials and Methods.

mice have less than $50 \mu \mathrm{g} / \mathrm{ml}$ of albuminuria using this assay, as previously reported $(n=12) .{ }^{16}$ Serum creatinine was measured by mass spectrometry as described. ${ }^{16}$

\section{Assessment of the Nephritogenic Immune Response}

Sandwich enzyme-linked immunosorbent assays (ELISAs) to measure the immune response to sheep IgG were

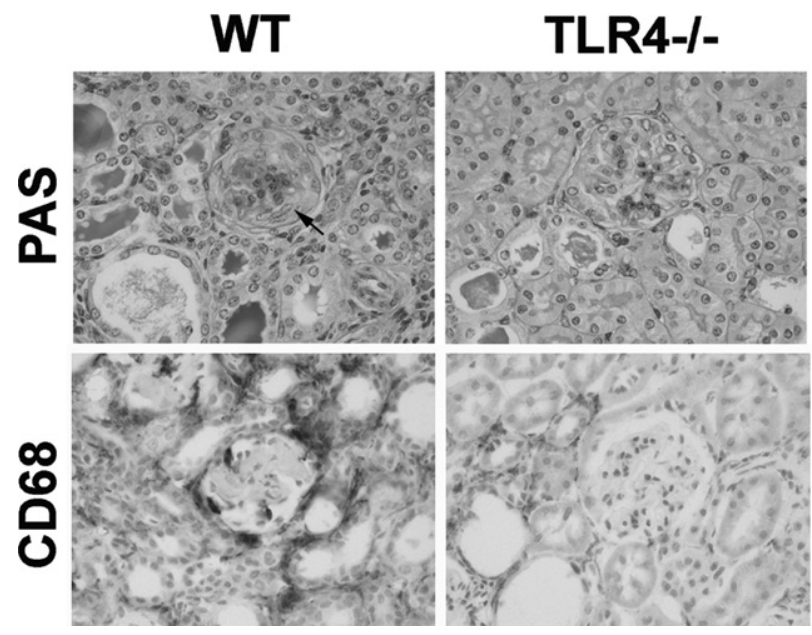

Figure 3. TLR4 stimulation at immunization exacerbates crescentic glomerulonephritis. Representative histology from wild-type and TLR4-deficient mice in the experiment shown in Figure 2. PAS stained sections showing a crescent and thrombosis in a wild-type but not a TLR4-deficient mouse. More macrophages were seen in the wild-type mice, as shown by CD68 immunoperoxidase staining. A crescent is shown by an arrow.

performed as before ${ }^{16}$ with modification for IgG2c. Detecting antibodies for IgG1, IgG2b, and IgG3 were from Southern Biotechnology (Birmingham, Alabama). The IgG2c antibody was from Bethyl Laboratories (Montgomery, TX). The relative concentrations were measured by using a standard curve that was constructed from pooled serum samples from mice that were immunized with sheep IgG. Normal serum from unimmunized mice served as a control. With disease induced according to protocol A, single cell suspensions were prepared from spleens at the end of the experiment. After red cell lysis, these were cultured at $10^{6} \mathrm{cells} / \mathrm{ml}$ for 5 days in X-Vivo 15 serum-free medium, containing 1\% glutamax (Invitrogen, Paisley, UK) and $0.1 \%$ mercaptoethanol, in the presence or absence of sheep $\mathrm{lgG}$ at $100 \mu \mathrm{g} / \mathrm{ml}$. The sheep IgG preparation was the same as that used for pre-immunization. Interleukin-4 and interferon- $\gamma$ were measured in supernatants by using ELISA kits (R\&D Systems, Oxford, UK) with limits of detection of $15.6 \mathrm{pg} / \mathrm{ml}$ and $31.2 \mathrm{pg} / \mathrm{ml}$, respectively.

\section{Bone Marrow Chimeras}

Chimeric mice were made by irradiation with a dose of 9 Gy and reconstitution with $5 \times 10^{6}$ donor bone marrow cells. Experiments were performed 10 weeks after this. Confirmation of chimerism was obtained by using realtime PCR for the neomycin resistance gene (present in TLR4-deficient mice) and glyceraldehyde-3-phosphate dehydrogenase, using genomic DNA extracted from peripheral blood, as described previously. ${ }^{11}$ DNA from a TLR4-deficient mouse was used as a standard, and results were expressed as a percentage of this value. This confirmed that bone marrow was at least 90\% donor derived in all mice tested ( $n=5$ per group). 
A

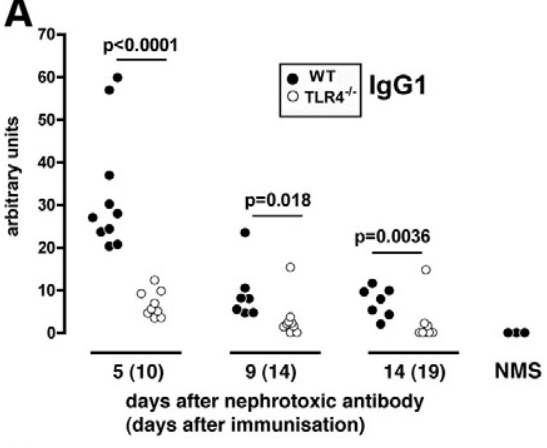

C

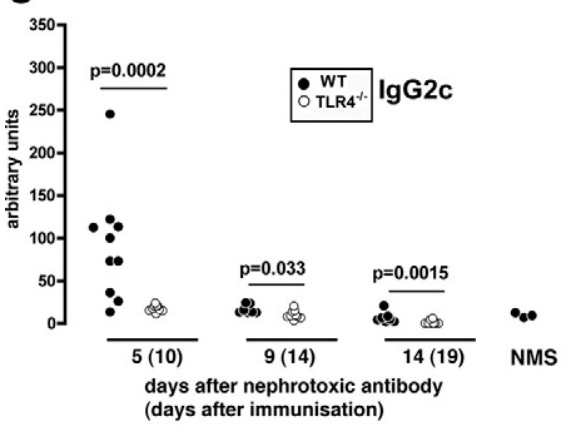

E

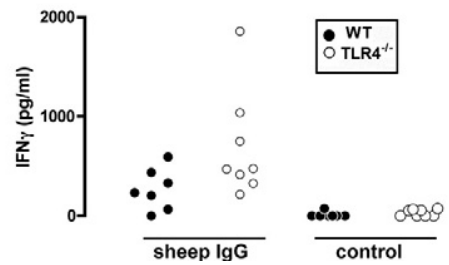

B
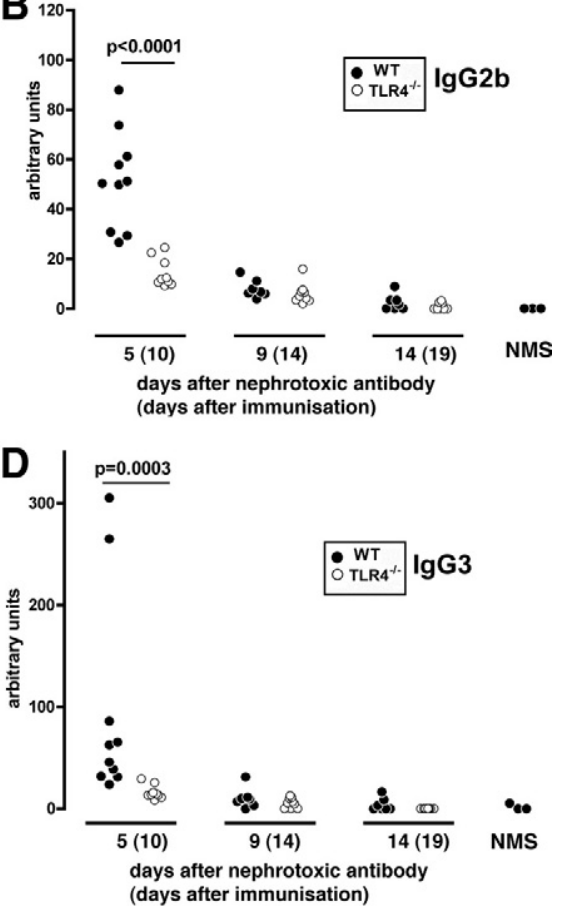

$\mathbf{F}$

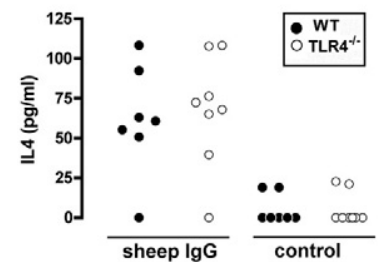

Figure 4. A-D: TLR4 stimulation at immunization augments the humoral immune response to sheep IgG. Subclass-specific ELISAs were performed on serum from wild-type and TLR4-deficient animals pre-immunized with sheep $\operatorname{IgC}$ and LPS in the experiment in Figure 2. Norma serum from three untreated mice was included as a control (NMS). Wild-type mice had an increase in all subclasses at day five when compared with TLR4-deficient mice. This difference was maintained at day nine and 14 for IgG1 and IgG2c. Each point is an individual mouse. $\mathbf{E}$ and F: Spleens were taken at the end of the experiment and cytokines were measured in supernatants from splenocytes restimulated in the presence of sheep IgG, with controls cultured without sheep IgG. The data demonstrate an antigen-specific response, but there were no significant differences between the groups. Each point is the mean of triplicate cultures from an individual mouse. At the end of the experiment $n=7$ (wild-type) and $n=8$ (TLR4-deficient) for all data sets, with additional surviving mice included at earlier time points.

\section{Statistics}

These were performed by using Graphpad Prism Software (GraphPad Software, Inc., La Jolla, CA). An unpaired Student's t-test was used to compare two groups. If the variances were significantly different, a logarithmic transformation was used. More than two groups were compared by using a Kruskall-Wallis test with Dunn's post test. Significance was taken as $P<0.05$.

\section{Results}

\section{Toll-Like Receptor 4 Stimulation at the Time of Immunization with Nephritogenic Antigen Exacerbates Glomerulonephritis}

We began by exploring the effect of TLR4 stimulation at priming on the adaptive immune response and disease severity in nephrotoxic nephritis to discover if our previously described results for TLR2 ${ }^{16}$ were generally applicable to other TLRs. Disease was induced by using protocol A (as shown in Figure 1 and described in Materials and Methods) in wild-type and TLR4-deficient mice. Mice were bled from the tail vein at days 5 and 9 after injection of NTS for assessment of the humoral immune response. Mice were placed in metabolic cages for assessment of albuminuria at days 7 and 14. This experiment showed that TLR4 stimulation at the time of priming with sheep IgG exacerbated disease. Figure 2, A-F, shows that there were more glomerular macrophages, crescents, and thrombosis in wild-type mice compared with TLR4-deficient mice. Neutrophil numbers were low, and there was no difference between the groups. These were $0.086 \pm$ 0.012 and $0.0725 \pm 0.026$ (mean \pm SEM cells per glomerular cross section) in wild-type and TLR4-deficient mice, respectively. There was also evidence of a functional effect as serum creatinine was higher in wild-type mice. There was no difference in albuminuria at day 7 , and there was higher albuminuria in TLR4-deficient mice at day 14 , which just reached statistical significance. This may have been a reflection of the renal failure developing in wild-type but not TLR4-deficient mice. Figure 3 shows representative histology from this experiment. These data established that crescentic glomerulonephritis is exacerbated by TLR4 stimulation when this occurs at the time of priming with an antigen that is then planted in the glomerulus.

\section{Toll-Like Receptor 4 Stimulation Augments the Nephritogenic Immune Response}

To explore the mechanism of more severe disease in wild-type mice compared with TLR4-deficient mice, we assessed the immune response to sheep IgG in the 


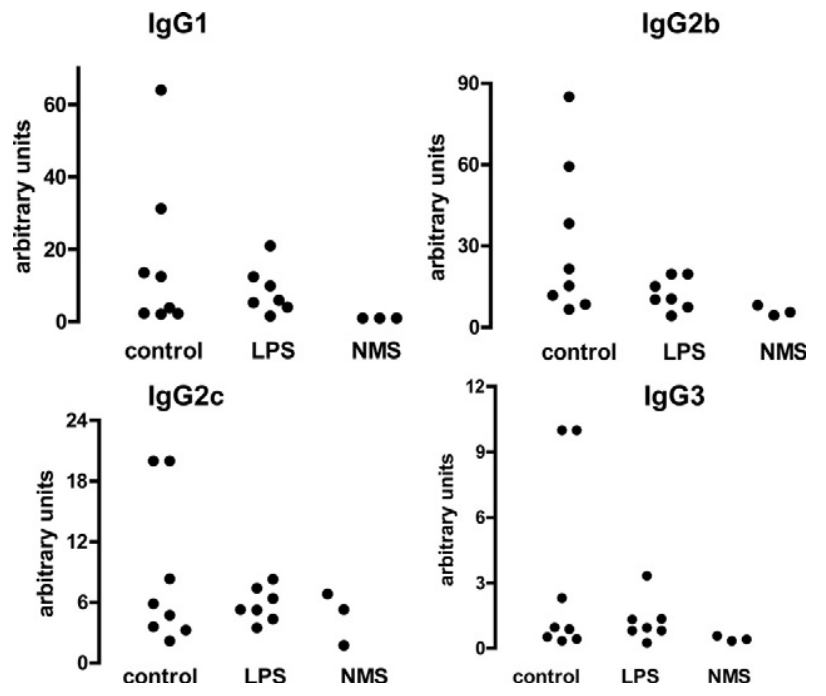

Figure 5. LPS given after disease induction does not affect the immune response to sheep IgG. Wild-type mice were given LPS or control at days five and seven after NTS and sacrificed at day 12 according to protocol B. Subclass-specific ELISAs were performed on serum, with normal mouse serum from three nonimmunized mice as a control (NMS). There were no significant differences. Each point is an individual mouse, with $n=7$ (LPS) and $n=8$ (control). Mice not surviving to day 12 were not included in the analysis and are listed in Materials and Methods.

above experiment. Using subclass-specific ELISAs, we analyzed serum at days 5, 9, and 14 after disease induction (corresponding to days 10, 14, and 19 after priming). As shown in Figure 4 (A-D), the peak response was seen at day 5, and there was an increase in all four IgG subclasses in wild-type compared with TLR4-deficient mice. The difference in IgG1 and IgG2c was maintained at later time points. To further assess any change in Th1 or Th2 skewing, we restimulated splenocytes with sheep IgG at the end of this experiment and measured interleukin-4 and interferon- $\gamma$ in supernatants. As shown in Figure 4 ( $E$ and $F$ ), there were no significant differences. We also performed immunofluorescence staining to assess glomerular deposition of mouse IgG subclasses and found increased IgG2c and IgG3 (see Supplemental Figure 1 at http://ajp.amjpathol.org). Because this is a less sensitive technique, the absence of a difference for IgG1 and IgG2b does not invalidate the ELISA data. Additional factors regarding interpretation of immunofluorescence data are discussed below. These data established that the increase in disease severity in wild-type mice compared with TLR4-deficient mice was accompanied by a global augmentation of the adaptive immune response to sheep IgG with no change in Th1 or Th2 skewing.

\section{Toll-Like Receptor 4 Stimulation after Disease Induction Does Not Affect the Nephritogenic Immune Response but Triggers Crescentic Disease}

We had established that TLR4 stimulation at the time of priming to the relevant antigen could augment disease by modulating the adaptive immune response. Our next aim was to establish an experimental protocol to test the
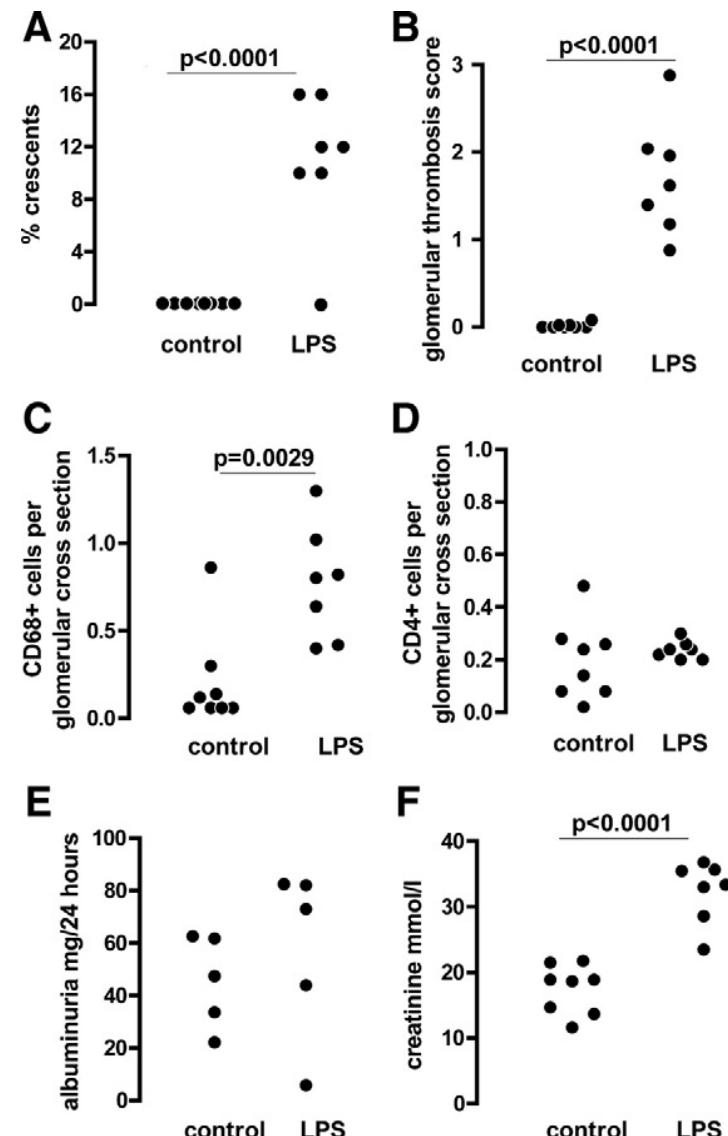

$\underline{p}<0.0001$

Figure 6. Nephrotoxic nephritis is exacerbated by LPS given after disease induction. Wild-type mice were given LPS or control at days 5 and 7 after NTS as described in Materials and Methods (protocol B). Data are from the same experiment as the ELISA data in Figure 4. A-D: There were significantly more crescents, thrombosis, and glomerular $\operatorname{CD} 68^{+}$macrophages but no difference in glomerular $\mathrm{CD}^{+}$cell numbers in mice given LPS compared with controls. E and F: There was no difference in albuminuria, but a higher serum creatinine in LPS treated mice. Each point is an individual mouse with $n=8$ (control) and $n=7$ (LPS).

effect of TLR4 stimulation later in disease development, with minimal effect on the nephritogenic immune response. We therefore induced disease by using protocol $\mathrm{B}$ (shown in Figure 1 and described in Materials and Methods) in wild-type C57BL/6 mice. We assessed the immune response to sheep IgG at the end of the experiment (day 14) by using subclass-specific ELISAs. As shown in Figure 5, no significant differences were seen between mice given LPS or control. Antigen-specific levels of all IgG subclasses were similar in both groups. We also performed immunofluorescence staining for IgG subclasses and performed a semiquantitative analysis of the intensity of glomerular staining. However, the distribution of staining did not follow the capillary wall as expected, and this was probably due to the large amount of thrombosis seen (Figure 6B). There was significantly more IgG2C and IgG3 present in mice given LPS (see Supplemental Figure 2 at http://ajp.amjpathol.org), but we were not confident that this was not the result of secondary trapping of these subclasses in more diseased glomeruli, and do not consider it a reliable measure of the immune response to sheep IgG. However, we acknowledge that we have not excluded some effect of LPS 


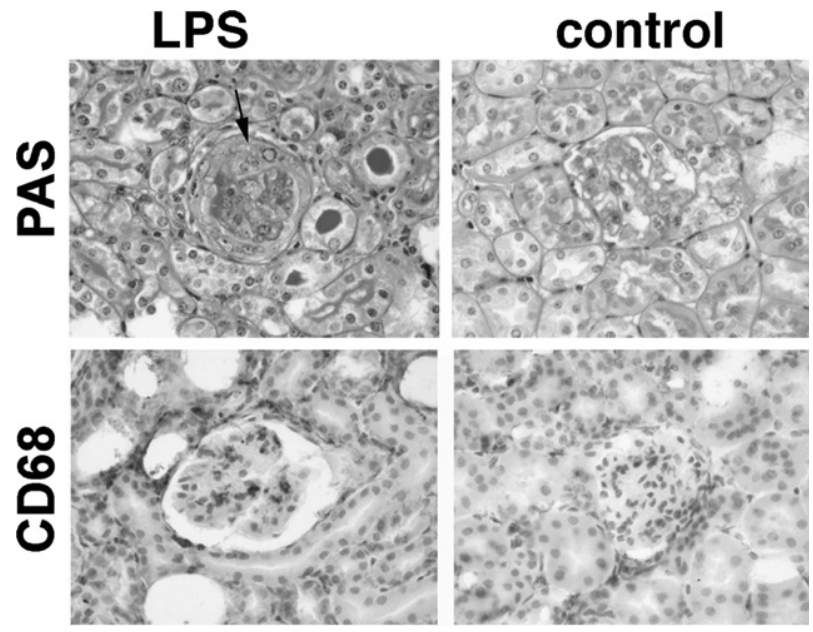

Figure 7. Nephrotoxic nephritis is exacerbated by LPS given after disease induction. Representative histology at day 14 from the experiment shown in Figure 5. This shows crescent formation and thrombosis in mice given LPS (left column) compared with controls (right column). There were also more glomerular $\mathrm{CD} 68^{+}$macrophages, but no difference in $\mathrm{CD}^{+}$cell numbers. A crescent is shown by an arrow.

administration on the nephritogenic immune response to sheep IgG.

Having established that LPS does not have a major effect on the immune response in this protocol, we then assessed the effect of LPS on disease in wild-type C57BL/6 mice in the above-described experiment. Administration of LPS led to more glomerular crescents, increased glomerular thrombosis, greater numbers of $\mathrm{CD}^{+}{ }^{+}$macrophages, but no difference in glomerular $\mathrm{CD}^{+}$cells as shown in Figure 6 (A-D). Representative histology is shown in Figure 7. Neutrophil numbers were low, and there was no difference between the groups. These were $0.045 \pm 0.023$ and $0.046 \pm 0.018$ (mean \pm SEM cells per glomerular cross section) in mice given control or LPS, respectively. To assess the functional effects of TLR stimulation, we measured albuminuria and serum creatinine with data shown in Figure 6 ( $E$ and $F$ ). Albuminuria data were only obtained from five mice per group due to technical issues. There was no difference in albuminuria, which was severe in both groups. However, serum creatinine was significantly higher in mice given LPS compared with control. These results established that LPS given after disease induction triggers crescentic disease with no detectable effect on the nephritogenic immune response. It should be noted that this was true even when the two mice with the most severe disease in the group given LPS were excluded form the analysis because they were sacrificed early.

\section{The Immune Response to the Nephritogenic Antigen Is Influenced by Bone Marrow but Not Renal Cell TLR4}

Having established that TLR4 stimulation triggered crescentic disease, we then explored the role of renal cell

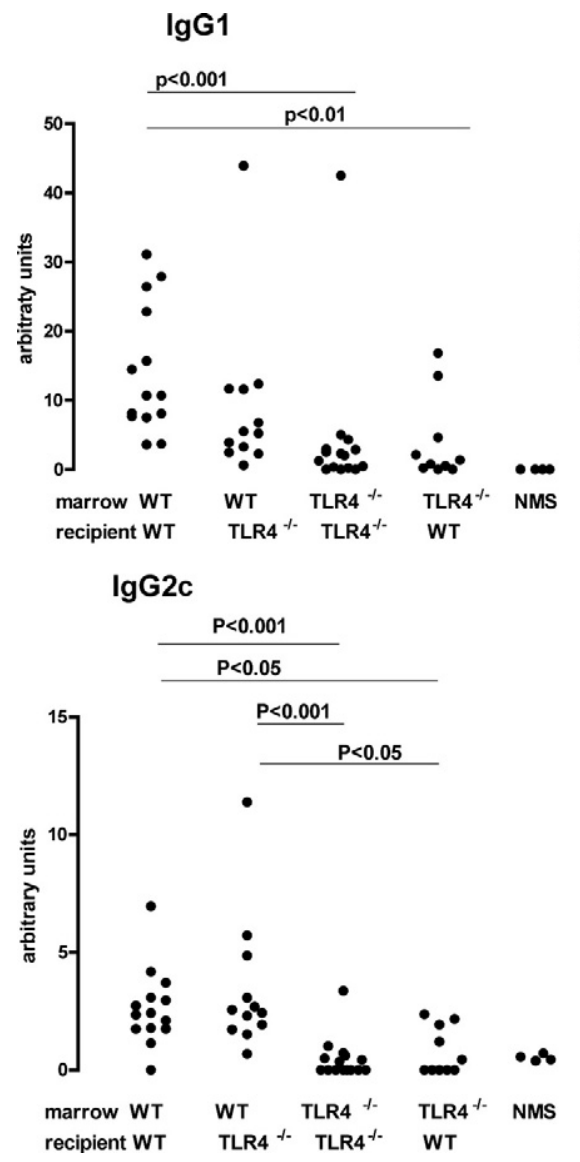

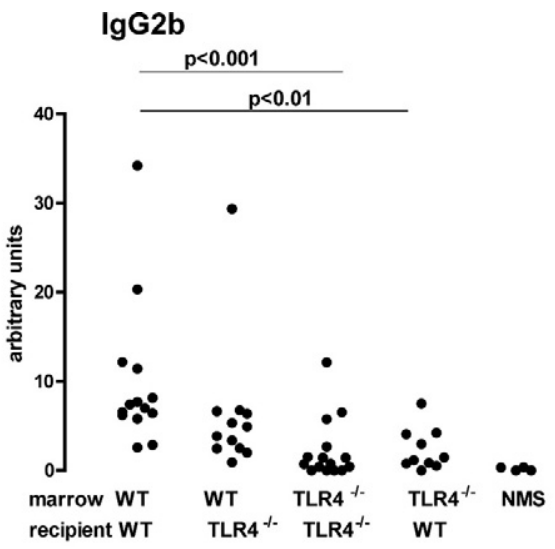

$\lg \mathrm{G} 3$

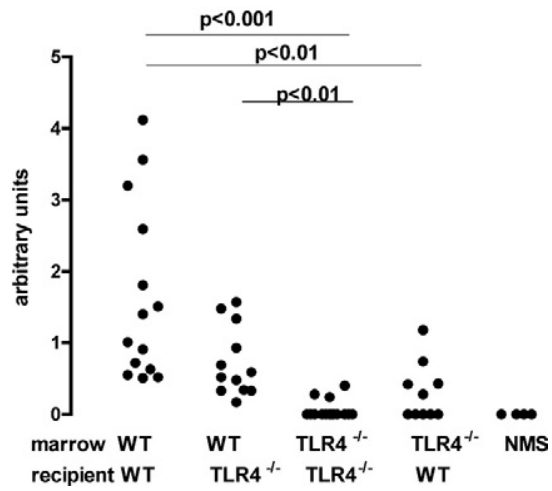

Figure 8. The immune response to sheep IgG in chimeric and sham chimeric mice with nephrotoxic nephritis given LPS after disease induction. Mice were given LPS at days five and seven after NTS and sacrificed at day 14 according to protocol B. Serum was analyzed by using subclass-specific ELISAs, with normal mouse serum from three nonimmunized mice as a control (NMS). Several differences were shown between mice with wildtype (WT) or TLR4 bone marrow with $P$ values shown for those that were significant. Importantly, there we no significant differences between mice that had the same bone marrow (between wildtype $\rightarrow$ wild-type and wild-type $\rightarrow$ TLR $^{->-}$, or between TLR4 ${ }^{-/-} \rightarrow \mathrm{TLR}^{-/-}$and TLR4 $4^{-/-} \rightarrow$ wild-type). Each point is an individual mouse with $n=14,15,13$, and 10 for wild-type $\rightarrow$ wild-type $\mathrm{TLR}^{-/-} \rightarrow \mathrm{TLR4}^{-/-}$, wild-type $\rightarrow \mathrm{TLR}^{-/}{ }^{-}$, and TLR4 $^{-\prime} \rightarrow$ wild-type mice, respectively. Mice not surviving to day 14 were not included in the analysis and are listed in Materials and Methods. 
A

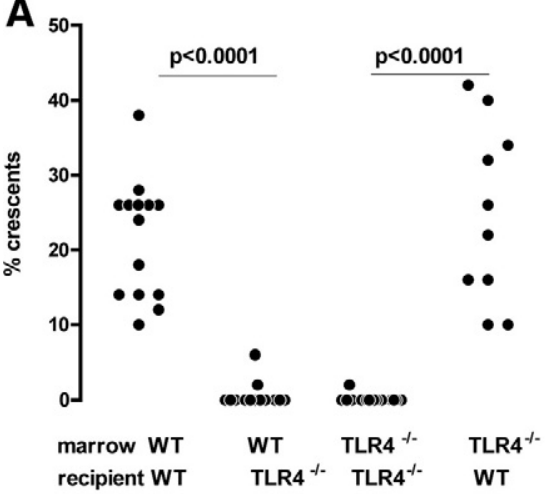

C

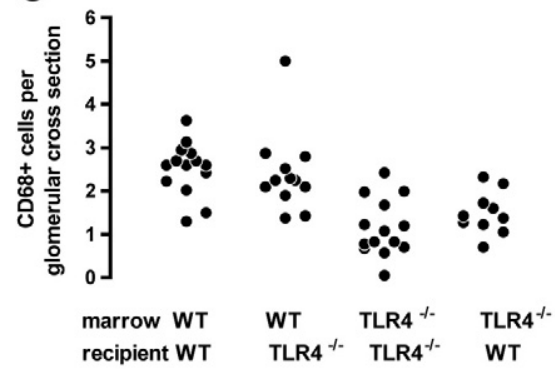

E

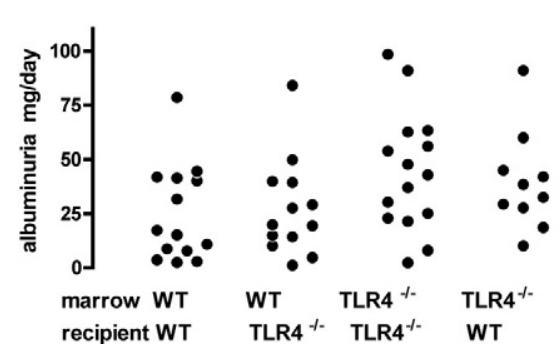

B

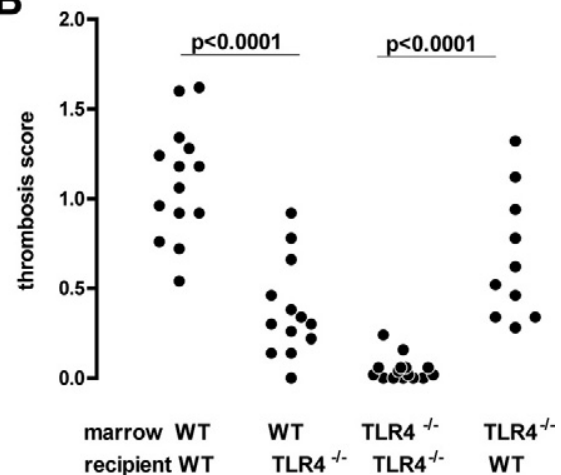

D

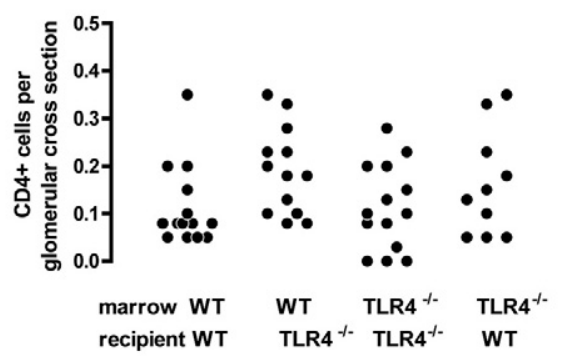

$\mathbf{F}$

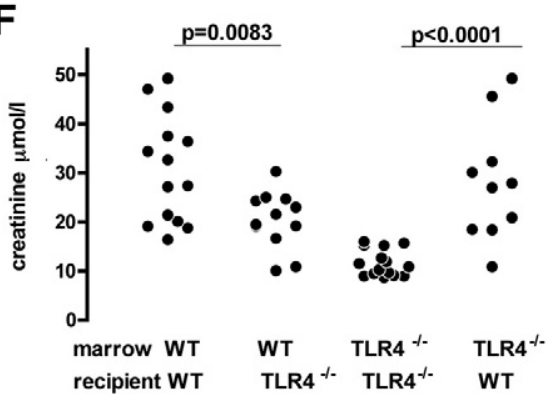

Figure 9. Stimulation of renal cell TLR4 exacerbates crescentic glomerulonephritis. Histological disease parameters assessed in chimeric and sham chimeric mice 14 days after disease induction with LPS or control given at days five and seven as described in Materials and Methods (protocol B). Statistical significance was only assessed between groups with the same bone marrow as discussed in the text. $\mathbf{A}$ and $\mathbf{B}$ : wild-type $\rightarrow$ wild-type (WT) mice had significantly more crescents and thrombosis than wild-type $\rightarrow$ TLR $^{-1-}$ animals. TLR $4^{-1-} \rightarrow$ wild-type mice had significantly more crescents and thrombosis than TLR4 $4^{-/-} \rightarrow$ TLR $^{-/-}$animals. C and D: There were no differences in glomerular $\mathrm{CD}^{+} 8^{+}$cell numbers when comparing mice with the same marrow, and no differences between any groups in glomerular $\mathrm{CD}^{+}$ cell numbers. E: There were no differences in albuminuria. F: wild-type $\rightarrow$ wild-type mice had significantly higher serum creatinines than wildtype $\rightarrow$ TLR $^{->-}$animals. TLR4 ${ }^{-/-} \rightarrow$ wild-type mice had significantly higher serum creatinines than TLR4 $4^{->} \rightarrow$ TLR4 $^{-1-}$ animals. Each point is an individual mouse with $n=14,15,13$, and 10 for wild-type $\rightarrow$ wild-type, TLR4 ${ }^{-/} \rightarrow$ TLR $^{-1-}$, wild-type $\rightarrow \mathrm{TLR}^{-/-}$, and TLR $4^{-/-} \rightarrow$ wild-type mice, respectively. Mice not surviving to day 14 were not included in the analysis and are listed in Materials and Methods.
TLR4 in this process. We constructed bone marrow chimeras by transplanting wild-type or TLR4-deficient bone marrow in TLR4-deficient or wild-type mice. Sham chimeras were constructed by transplanting wild-type marrow into wild-type mice, or TLR4-deficient marrow into TLR4deficient mice. We induced disease in these four groups of chimeric or sham chimeric mice by using the same protocol B that had been used in wild-type mice as described above, with all mice receiving intraperitoneal LPS at days 5 and 7 , with mice sacrificed at day 14. We began by analyzing the nephritogenic immune response with subclass-specific ELISAs as shown in Figure 8. We found that there was a diminished immune response in mice that had TLR4-deficient marrow compared with mice with wild-type marrow. This was surprising since we had found no difference between wild-type mice given PBS or control using this protocol (Figure 4), and this is considered in the discussion. These differences varied for the subclasses, and any statistically significant differences are indicated in Figure 4. The important point to note is that there were no differences between mice with the same bone marrow for any of the subclasses. The question that we set out to address in these studies is the role of renal cell TLR stimulation on crescentic disease in the absence of an effect on the adaptive immune response. To achieve this it was necessary to compare groups of mice with the same immune response to sheep IgG. Therefore, in analyzing disease parameters in this experiment, we restricted comparisons to those between mice with the same bone marrow and hence the same immune response. That is, wild-type $\rightarrow$ wild-type and wild-type $\rightarrow \mathrm{TLR}^{-1-}$ mice were compared, and $\mathrm{TLR}^{-1-} \rightarrow \mathrm{TLR}^{-1-}$ and TLR4 ${ }^{-1-} \rightarrow$ wild-type mice were compared.

\section{Renal Cell Toll-Like Receptor 4 Stimulation Triggers Crescentic Disease}

Having established that there was no difference in the immune response for chimeric or sham chimeric mice with the same marrow, we assessed the disease parameters in this experiment as shown in Figure 9, A-F. When wild-type $\rightarrow$ wild-type and wild-type $\rightarrow \mathrm{TLR}^{-1-}$ mice were compared, there was significantly less thrombosis and crescent formation in the latter. This means that, in 


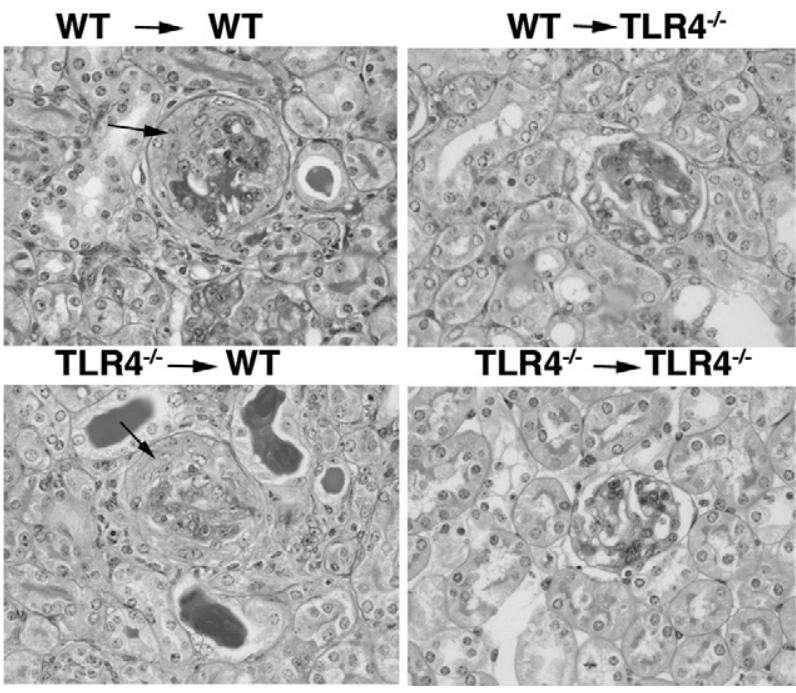

Figure 10. Stimulation of renal cell TLR 4 exacerbates disease. Representative histology from the experiment in Figure 8, showing crescents in wildtype $\rightarrow$ wild-type (WT) and TLR $4^{-/} \rightarrow$ wild-type mice, with a degree of thrombosis in all groups except TLR $4^{-/-} \rightarrow \mathrm{TLR}^{-/-}$animals. Crescents are shown by arrows

the setting of LPS administration after disease induction, the lack of renal cell TLR4 protects from crescent formation and thrombosis. In fact, crescent formation was practically absent in wild-type $\rightarrow \mathrm{TLR}^{-1-}$ mice. Despite the increase in crescent formation and thrombosis, numbers of glomerular macrophages and CD4 positive $\mathrm{T}$ cells were the same in wild-type $\rightarrow$ wild-type and wild-type $\rightarrow \mathrm{TLR}^{-1-}$ mice. When $\mathrm{TLR}^{-1-} \rightarrow \mathrm{TLR}^{-1-}$ and $\mathrm{TLR}^{-1-} \rightarrow$ wild-type mice were compared, we found that there was significantly more thrombosis and crescent formation in the latter. This means that, in the setting of LPS administration after disease induction, the presence of renal cell TLR4 promotes crescent formation and thrombosis, with crescent formation practically absent in TLR4 ${ }^{-1-} \rightarrow \mathrm{TLR}^{-1-}$ mice. Despite the increase in crescent formation and thrombosis, numbers of glomerular macrophages and CD4 positive T cells were the same in $\mathrm{TLR}^{-1-} \rightarrow \mathrm{TLR}^{-1-}$ and $\mathrm{TLR}^{-1-} \rightarrow$ wild-type mice. Neutrophil numbers were low, and there was no difference between the groups. These were $0.13 \pm$ $0.025,0.08 \pm 0.031,0.16 \pm 0.027$, and $0.07 \pm 0.17$ (mean \pm SEM cells per glomerular cross section) in

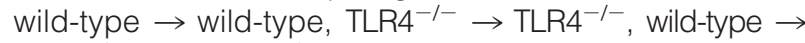
$\mathrm{TLR}^{-1-}$, and TLR4 ${ }^{-1-} \rightarrow$ wild-type groups, respectively. Representative histology from these experiments in chimeric mice is shown in Figure 10. There were no differences in albuminuria when either wild-type $\rightarrow$ wild-type and wildtype $\rightarrow \mathrm{TLR}^{-1-}$ mice, or $\mathrm{TLR}^{-1-} \rightarrow \mathrm{TLR}^{-1-}$ and $\mathrm{TLR}^{-l-} \rightarrow$ wild-type mice were compared. In contrast, differences in serum creatinine mirrored the changes seen in glomerular thrombosis and crescent formation demonstrating an important functional consequence of these morphological changes. Wild-type $\rightarrow$ wild-type mice had significantly higher serum creatinines than wildtype $\rightarrow$ TLR4 $^{-1-}$ mice. Likewise TLR4 ${ }^{-1-} \rightarrow$ wild-type mice had higher serum creatinines than $\mathrm{TLR}^{-1-} \rightarrow$ $\mathrm{TLR}^{-1-}$ mice. To examine the relationship between throm- bosis and crescent formation, we plotted these as shown in Supplemental Figure 3 at $h$ ttp://ajp.amjpathol.org. Although several mice in the wild-type $\rightarrow \mathrm{TLR}^{-1-}$ group showed a degree of thrombosis comparable to that seen in wildtype $\rightarrow$ wild-type and TLR4 ${ }^{-1-} \rightarrow$ wild-type mice, they did not develop crescents. There was a correlation between crescent formation and thrombosis in $\mathrm{TLR}^{-1-} \rightarrow$ wild-type mice but not in the other two groups individually, or when the data from all three groups were analyzed together. This suggested that thrombosis itself was not sufficient for crescent formation, and that this resulted from a different and specific effect of TLR4 stimulation on renal cells. Overall, these data demonstrate that renal cell TLR4 stimulation can trigger glomerular thrombosis, crescent formation, and renal failure.

\section{Discussion}

These results extend our previously published findings showing that TLR2 stimulation augments the nephritogenic immune response without causing a change in the Th1 versus Th2 balance. ${ }^{16}$ We have therefore established a general mechanism whereby either TLR2 or TLR4 stimulation can exacerbate crescentic glomerulonephritis. We did not demonstrate a difference in cellular immunity as evidenced by glomerular $\mathrm{CD}^{+}$cell numbers or the cytokines released by restimulated splenocytes. The precise mechanism by which humoral immunity is augmented could therefore be via direct effects on $\mathrm{B}$ cells, or via stimulation of antigen presenting cells, which would then prime $T$ helper cells and augment the humoral response. These possibilities are not mutually exclusive and could both apply. It should be noted that TLR4 may be stimulated by both exogenous microbial ligands or by endogenous ligands. ${ }^{18}$ In this experiment, TLR4 stimulation would have been the result of administered LPS and also any contribution from endogenous ligands present in normal mice. However, the comparison of wild-type and TLR4-deficient mice gives a clear assessment of the role of TLR4 in this context, since the presence or absence of TLR4 is the only difference between the groups.

The demonstration of a crucial role for renal cell TLR4 in the pathogenesis of crescentic disease is probably the most unexpected result that we report here. The interpretation of the chimera experiments is complicated by differences in the immune response between groups that had wild-type or TLR4 ${ }^{-1-}$ bone marrow. Further studies (data not shown) have suggested that the effect on the immune response may be due to the sheep serum having some TLR stimulatory activity at the time of initial exposure to sheep $\operatorname{lgG}$, despite the fact that the endotoxin content of the serum used was $<0.5 \mathrm{eU} / \mathrm{ml}$. As discussed above, endogenous ligands for TLR4 may also be present in normal mice and could contribute to the differences in the immune response seen. Nevertheless, when mice with the same marrow were compared, our data clearly show that renal cell TLR4 stimulation can lead to crescentic glomerulonephritis. The effect on crescent formation was striking. Of the four chimeric or sham 
chimeric groups, only the two groups with renal cell TLR4 (wild-type recipients) developed crescents, with virtually none in the other two groups. Despite the diminished immune response in mice with $\mathrm{TLR} 4^{-/-}$marrow, renal cell TLR4 stimulation (in TLR4 ${ }^{-1-} \rightarrow$ wild-type mice) was sufficient to trigger crescentic disease in these animals.

There were no differences in albuminuria between chimeric and sham chimeric groups. We had also failed to detect differences in albuminuria in experiments that did not involve chimeras (Figures 2 and 6) and it is possible that the reduction in renal function as evidenced by a raised serum creatinine decreased the measured albuminuria in mice with increased disease. In addition, the albuminuria was variable, and in the case of the bone marrow chimera experiments, this could be due to a variable degree of chimerism between animals, although this would not explain the variability in wild-type $\rightarrow$ wildtype and $\mathrm{TLR}^{-1-} \rightarrow \mathrm{TLR}^{-1-}$ groups. The differences in crescent count suggest a high degree of chimerism, and we demonstrated this by using quantitative PCR on peripheral blood. However, this may not be as accurate a method as assessment at the cellular level with flow cytometry.

When mice with the same marrow and immune response were compared, there were no differences in glomerular macrophages, and all four groups had similar numbers of glomerular CD4 positive cells. The differences in macrophage number and thrombosis between $\mathrm{TLR}^{-1-} \rightarrow$ wild-type and wild-type $\rightarrow$ wild-type, or between $\mathrm{TLR}_{4}{ }^{-1-} \rightarrow \mathrm{TLR}_{4}{ }^{-1-}$ and wild-type $\rightarrow \mathrm{TLR}^{-1-}$ mice may have been a reflection of differences in the immune response in addition to differences in downstream effects on leukocytes. Although we have emphasized the fact that the two groups with TLR4-deficient marrow had a lesser immune response to sheep IgG than those with wild-type marrow, this did not influence the formation of crescents when renal cell TLR4 was present. This occurred to a similar extent in TLR4 ${ }^{-l-} \rightarrow$ wild-type and wild-type $\rightarrow$ wild-type mice despite a lesser immune response to sheep IgG in the former. The differences in crescent formation between groups with the same marrow occurred despite similar macrophage numbers, suggesting that macrophage infiltration was not sufficient to cause crescent formation. Although mice in the wild-type $\rightarrow \mathrm{TLR}^{-1-}$ group developed less thrombosis than mice in wild-type $\rightarrow$ wild-type and TLR $4^{-1-} \rightarrow$ wildtype groups, there was considerable overlap. Some mice in the wild-type $\rightarrow \mathrm{TLR}^{-/-}$group had a degree of thrombosis similar to that of these other groups as illustrated in

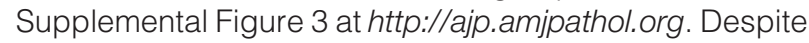
this they did not develop crescents. This suggests that thrombosis itself does not cause and is not sufficient for crescent formation.

There are previous reports of TLR stimulation of intrinsic renal cells causing kidney disease. A bone marrow chimera approach has defined a role for both TLR2 and TLR4 on renal cells in ischemia reperfusion injury, ${ }^{8-10}$ and also shown a role for renal cell TLR4 in a model of infection. ${ }^{7}$ In contrast, LPS-induced acute kidney injury in mice was not dependent on renal cell TLR4, as evidenced by studies with transplanted TLR4-deficient and wild-type kidneys. ${ }^{19}$ These studies have shown that in some experimental systems, renal tubular cell TLR4 may be important. Our own work in heterologous nephrotoxic nephritis has established that renal cell TLR2 or TLR4 stimulation may also be important in glomerular disease. It exacerbates the neutrophil influx and proteinuria induced by passively administered glomerular-binding antibody..$^{11,12}$ The current study differs from our previous reports because, unlike heterologous nephrotoxic nephritis, the autologous phase is a model in which progressive disease occurs, and histological features closely resemble those seen in human glomerulonephritis.

In a previous study we have localized TLR4 in the glomerulus by using in situ hybridization. ${ }^{12}$ We showed that parietal and visceral epithelial cells express TLR4, and there was also mesangial expression, with TLR4dependent chemokine release from cultured mesangial cells. TLR3 has been found on mesangial cells and implicated in the pathogenesis of murine lupus, ${ }^{20}$ although in vivo evidence of a role was not determined through the use of bone-marrow chimeras. More recently other investigators have detected protein with immunohistochemical staining for TLR4 reported to be on podocytes in normal mouse glomeruli. ${ }^{21}$ Murine glomerular endothelial cells and podocytes both express TLR4, ${ }^{22}$ and cultured podocytes have been shown to express TLR4 and to undergo phenotypic changes including cytoskeletal reorganization and B7.1 expression in response to TLR4 stimulation. ${ }^{23} \mathrm{~A}$ further report demonstrated that podocytes express a range of chemokines in response to TLR4 stimulation. ${ }^{21}$ However, TLR4 has been localized to the glomerular endothelium rather than podocytes in frozen rat kidney, ${ }^{24}$ and it seems likely that the findings would be the same in mice. Both Myd88 and TRIF dependent TLR4 signaling has been shown in murine mesangial cells, although TRIF deficient mice developed nephrotoxic nephritis to a similar degree to wild-type mice. ${ }^{25}$ However, a different disease induction protocol to the present study was used, with priming in Freund's adjuvant and LPS was not given during disease development. We acknowledge that a limitation of our study is that we do not define the renal cell responsible for the TLR4-mediated effects on crescent formation. Because resident renal leukocytes such as dendritic cells are radiosensitive, the cell responsible will almost certainly be parenchymal. Crescent formation is thought to occur when the glomerular endothelium is damaged, leading to the entry of blood components into Bowman's space. Whether this is a direct effect of endothelial cell TLR4 stimulation or is mediated via TLR4-dependent effects of TLR4 stimulation on podocytes or mesangial cells remains to be defined in future studies. Such studies would require cell-specific manipulation of TLR4 to provide definitive answers.

We have focused on one receptor, namely TLR4, in the current study. We accept that this is a simplification, since in the context of infection, pathogens would be expected to activate a number of TLRs and Nod-like receptors simultaneously providing a more wide-ranging response. However, by limiting our study to TLR4, we have been able to reveal the multiple facets of the response to even this single receptor. The complexity of our 
experimental system is reflected in the fact that we have shown that TLR4 stimulation can both augment the nephritogenic immune response and have direct effects on renal cells. Our findings therefore represent a novel observation on the interactions between the innate and adaptive arms of the immune system in causing glomerulonephritis. We show the integration of TLR stimulation on both the adaptive and innate immune systems in a complex and relevant disease model, with crucial effects of TLR4 stimulation on renal cells in the development of pathology.

\section{Acknowledgments}

We are grateful to Shizuo Akira for providing TLR4-deficient mice. We acknowledge the support of the MRC Centre for Transplantation.

\section{References}

1. Poltorak A, He X, Smirnova I, Liu MY, Van Huffel C, Du X, Birdwell D, Alejos E, Silva M, Galanos C, Freudenberg M, Ricciardi-Castagnoli P, Layton B, Beutler B: Defective LPS signaling in C3H/HeJ and C57BL/ 10ScCr mice: mutations in TIr4 gene. Science 1998, 282:2085-2088

2. Takeuchi O, Hoshino K, Kawai T, Sanjo H, Takada H, Ogawa T, Takeda K, Akira S: Differential roles of TLR2 and TLR4 in recognition of gram-negative and gram-positive bacterial cell wall components. Immunity 1999, 11:443-451

3. Takeda K, Akira S: Toll-like receptors in innate immunity. Int Immunol 2005, 17:1-14

4. Zarember KA, Godowski PJ: Tissue expression of human Toll-like receptors and differential regulation of Toll-like receptor mRNAs in leukocytes in response to microbes, their products, and cytokines. J Immunol 2002, 168:554-561

5. Andonegui G, Bonder CS, Green F, Mullaly SC, Zbytnuik L, Raharjo E, Kubes P: Endothelium-derived Toll-like receptor-4 is the key molecule in LPS-induced neutrophil sequestration into lungs. J Clin Invest 2003, 111:1011-1020

6. Schilling JD, Mulvey MA, Vincent CD, Lorenz RG, Hultgren SJ: Bacterial invasion augments epithelial cytokine responses to Escherichia coli through a lipopolysaccharide-dependent mechanism. J Immunol 2001, 166:1148-1155

7. Patole PS, Schubert S, Hildinger K, Khandoga S, Khandoga A, Segerer S, Henger A, Kretzler M, Werner M, Krombach F, Schlondorff $\mathrm{D}$, Anders HJ: Toll-like receptor-4: renal cells and bone marrow cells signal for neutrophil recruitment during pyelonephritis. Kidney Int 2005, 68:2582-2587

8. Wu H, Chen G, Wyburn KR, Yin J, Bertolino P, Eris JM, Alexander SI, Sharland AF, Chadban SJ: TLR4 activation mediates kidney ischemia/reperfusion injury. J Clin Invest 2007, 117:2847-2859

9. Shigeoka AA, Holscher TD, King AJ, Hall FW, Kiosses WB, Tobias PS, Mackman N, McKay DB: TLR2 is constitutively expressed within the kidney and participates in ischemic renal injury through both MyD88-dependent and -independent pathways. J Immunol 2007, 178:6252-6258
10. Leemans JC, Stokman G, Claessen N, Rouschop KM, Teske GJ, Kirschning CJ, Akira S, van der Poll T, Weening JJ, Florquin S: Renal-associated TLR2 mediates ischemia/reperfusion injury in the kidney. J Clin Invest 2005, 115:2894-2903

11. Brown HJ, Lock HR, Sacks SH, Robson MG: TLR2 stimulation of intrinsic renal cells in the induction of immune-mediated glomerulonephritis. J Immunol 2006, 177:1925-1931

12. Brown HJ, Lock HR, Wolfs TG, Buurman WA, Sacks SH, Robson MG: Toll-like receptor 4 ligation on intrinsic renal cells contributes to the induction of antibody-mediated glomerulonephritis via CXCL1 and CXCL2. J Am Soc Nephrol 2007, 18:1732-1739

13. Cochrane CG, Unanue ER, Dixon FJ: A Role of polymorphonuclear leukocytes and complement in nephrotoxic nephritis. J Exp Med 1965, 122:99-119

14. Unanue ER, Dixon FJ: Experimental glomerulonephritis: the autologous phase of nephrotoxic seum nephritis. J Exp Med 1965, 121:715-725

15. Tipping PG, Kitching AR: Glomerulonephritis. Th1 and Th2: what's new? Clin Exp Immunol 2005, 142:207-215

16. Brown HJ, Sacks SH, Robson MG: Toll-like receptor 2 agonists exacerbate accelerated nephrotoxic nephritis. J Am Soc Nephrol 2006, 17:1931-1939

17. Fu Y, Xie C, Chen J, Zhu J, Zhou H, Thomas J, Zhou XJ, Mohan C: Innate stimuli accentuate end-organ damage by nephrotoxic antibodies via Fc receptor and TLR stimulation and IL-1/TNF-alpha production. J Immunol 2006, 176:632-639

18. Robson MG: Toll-like receptors and renal disease. Nephron Exp Nephrol 2009, 113:e1-e7

19. Cunningham PN, Wang Y, Guo R, He G, Quigg RJ: Role of Toll-like receptor 4 in endotoxin-induced acute renal failure. J Immunol 2004, 172:2629-2635

20. Patole PS, Grone HJ, Segerer S, Ciubar R, Belemezova E, Henger A, Kretzler M, Schlondorff D, Anders HJ: Viral double-stranded RNA aggravates lupus nephritis through toll-like receptor 3 on glomerular mesangial cells and antigen-presenting cells. J Am Soc Nephrol 2005, 16:1326-1328

21. Banas MC, Banas B, Hudkins KL, Wietecha TA, lyoda M, Bock E, Hauser P, Pippin JW, Shankland SJ, Smith KD, Stoelcker B, Liu G, Grone HJ, Kramer BK, Alpers CE: TLR4 links podocytes with the innate immune system to mediate glomerular injury. J Am Soc Nephrol 2008, 19:704-713

22. Pawar RD, Castrezana-Lopez L, Allam R, Kulkarni OP, Segerer S, Radomska E, Meyer TN, Schwesinger CM, Akis N, Grone HJ, Anders $\mathrm{HJ}$ : Bacterial lipopeptide triggers massive albuminuria in murine lupus nephritis by activating toll-like receptor 2 at the glomerular filtration barrier. Immunology 2009, 128:e206-e221

23. Reiser J, von Gersdorff G, Loos M, Oh J, Asanuma K, Giardino L, Rastaldi MP, Calvaresi N, Watanabe H, Schwarz K, Faul C, Kretzler M, Davidson A, Sugimoto H, Kalluri R, Sharpe AH, Kreidberg JA, Mundel P: Induction of B7-1 in podocytes is associated with nephrotic syndrome. J Clin Invest 2004, 113:1390-1397

24. El-Achkar TM, Huang X, Plotkin Z, Sandoval RM, Rhodes GJ, Dagher PC: Sepsis induces changes in the expression and distribution of Toll-like receptor 4 in the rat kidney. Am J Physiol Renal Physiol 2006, 290:F1034-F1043

25. Lichtnekert J, Vielhauer V, Zecher D, Kulkarni OP, Clauss S, Segerer S, Hornung V, Mayadas TN, Beutler B, Akira S, Anders HJ: Trif is not required for immune complex glomerulonephritis: dying cells activate mesangial cells via TIr2/Myd88 rather than TIr3/Trif. Am J Physiol Renal Physiol 2009, 296:F867-F874 